\title{
2. SYNOPSIS
}

\begin{tabular}{|l|l|}
\hline Name of Sponsor: I.R.I.S., 50 rue Carnot - 92284 Suresnes Cedex - France & $\begin{array}{l}\text { National } \\
\text { Authority Use only) }\end{array}$ \\
\hline Test drug & \\
Name of Finished Product: & \\
Not applicable & \\
Name of Active Ingredient: & \\
S 78454 (abexinostat) & Page: \\
\hline Individual Study Table Referring to Part of the Dossier & Volume: \\
\hline Title of study: Phase I dose-escalation study of S 78454 (HDACi) p.o. in combination with FOLFOX in \\
patients with locally advanced or metastatic digestive cancer. \\
Protocol No.: CL1-78454-006. \\
EudraCT No.: 2010-022369-89. \\
The description of the study protocol given hereafter includes the modifications of the two substantial \\
amendments to the protocol. \\
\hline International coordinator: \\
\hline
\end{tabular}

Study centres:

Three centres located in three countries included 32 patients: one centre in France included 10 patients, one centre in Belgium included 11 patients and one centre in Italy included 11 patients.

\section{Publication (reference):}

Not applicable.

\section{Studied period:}

Initiation date: 05 December 2011 (date of first visit first patient).

Phase of development of the study:

Completion date: 06 November 2014 (date of last visit last patient) Phase I

Amendment No. 1 applicable in all countries postponed the completion date from May 2013 to September 2014. Amendment No. 2 applicable in all countries postponed the completion date from September 2014 to December 2014.

\section{Objectives:}

\section{Primary Objectives}

- To assess the safety and tolerability of S 78454 given per os (p.o.) in combination with FOLinic acid (leucovorin)/Fluorouracil/OXaliplatin (FOLFOX) in patients with measurable or evaluable, refractory, metastatic colorectal cancer (mCRC) or locally advanced or metastatic gastric or pancreatic cancer in terms of the Maximum Tolerated Dose (MTD) and the Dose-Limiting Toxicities (DLTs).

- To establish the Recommended Phase II Dose (RP2D) of S 78454 given p.o. in combination with FOLFOX.

\section{Secondary Objectives}

- To determine the pharmacokinetic (PK) profile of S 78454 p.o. and its main metabolites, alone and in association with FOLFOX.

- To measure the tumour morphological response to S 78454 p.o. in association with FOLFOX using the revised RECIST guideline (v1.1).

- To evaluate the tumour metabolic response by $\left[{ }^{18} \mathrm{FDG}\right]-\mathrm{PET} / \mathrm{CT}$-scan.

- To evaluate the progression free survival (PFS).

- To determine the PK profile of 5-fluorouracil (5-FU) and oxaliplatin in association with S 78454.

- Optional part: pharmacogenetic analysis of the metabolism of S 78454 on blood sample. 


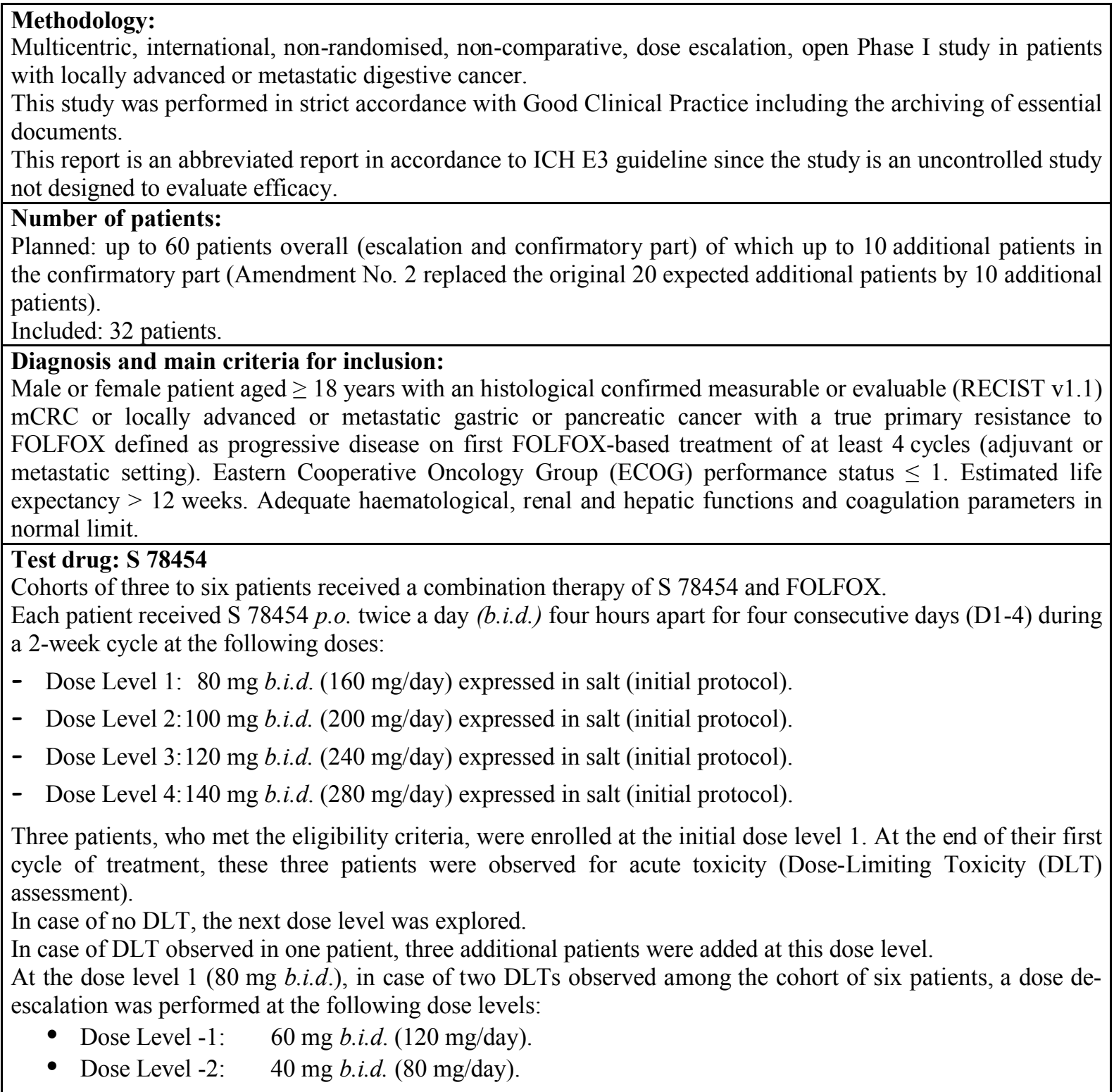

In case of no MTD reached at the dose level 4, additional cohorts were to be enrolled. The doses were to be increased by $20 \mathrm{mg}$ per cohort until the MTD was reached.

Due to technical manufacturing constraints, therapeutic units with dosages expressed in base were used after the start of the study (Amendment No 1) and these new therapeutic units had $9 \%$ more drug substance than the previous therapeutic units with dosages expressed in salt.

When Amendment No 1 was issued, the dose level $160 \mathrm{mg}$ b.i.d. expressed in salt was on going. In the first three patients, one DLT appeared (increase of bilirubin). Three other patients were included in this cohort. The new escalation scheme was as follows:

- At the end of this cohort, in case of no DLT in six patients, the dose level was to be increased to $160 \mathrm{mg}$ b.i.d expressed in base (i.e. $174.4 \mathrm{mg}$ b.i.d. expressed in salt) instead of $180 \mathrm{mg}$ b.i.d. expressed in salt and then additional cohorts were to be enrolled, increasing the dose by $20 \mathrm{mg}$ (expressed in base) per cohort until the MTD was reached.

- In case of two DLTs in these six patients, the MTD was $160 \mathrm{mg}$ b.i.d. expressed in salt and the lower dose level was $140 \mathrm{mg}$ b.i.d expressed in salt. In order to well define the recommended dose for the confirmatory part, a new cohort at dose level $140 \mathrm{mg}$ b.i.d. expressed in base was to be tested. If there was no DLT on three patients or one DLT on six patients, the $140 \mathrm{mg}$ b.i.d. expressed in base was to be considered as the recommended dose and used for the confirmatory part. If two DLTs appeared in the cohort, the $120 \mathrm{mg}$ b.i.d. expressed in base was the recommended dose. 


\section{Test drug: S 78454 (Cont'd)}

All patients, in a given cohort, required to have completed one 2-week cycle of therapy before the next cohort started.

After cycle 1, intra-patient dose reduction was permitted. Patients with toxicities other than grade $\geq 3$ QT interval corrected for heart rate (QTc) prolongation (determined by a central reading centre) could be retreated at the next lower dose when:

- The toxicity had resolved to baseline level (the level before treatment administration).

- Or according to the investigator's opinion, the patient conditions had improved enough to continue the treatment with respect to safety criteria.

The Recommended Phase II Dose (RP2D) was defined as one dose step below MTD.

Confirmatory part:

Up to 10 additional patients, with $\mathrm{mCRC}$ or locally advanced or metastatic gastric or pancreatic cancer refractory to FOLFOX, were to be included at the defined RP2D to better assess the acute and cumulative toxicity profile.

Batch numbers:

S $7845420 \mathrm{mg}$ with strength expressed in salt, hereafter abbreviated as (S): L0036983.

S $7845420 \mathrm{mg}$ with strength expressed in base, hereafter abbreviated as (B): L0039582, L0053415.

S $78454100 \mathrm{mg}$ with strength expressed in base, hereafter abbreviated as (B): L0039583, L0053416.

Other drugs: FOLFOX was administered on D3-5 of each cycle, two hours after the first S 78454 intake (H0):

- H2-H4: oxaliplatin $85 \mathrm{mg} / \mathrm{m}^{2}$ i.v. infusion on D3.

- H2-H4: calcium folinate $400 \mathrm{mg} / \mathrm{m}^{2}$ i.v. infusion on D3.

- H4-H50: 5-fluorouracil (5-FU) $2400 \mathrm{mg} / \mathrm{m}^{2}$ continuous i.v. infusion on D3-5.

\section{Comparator (Reference product and/or placebo):}

None

\section{Duration of treatment:}

Each patient was to receive at least four cycles of treatment (S 78454 + FOLFOX) except in case of safety concerns. The maximum number of cycles was at the discretion of the investigator.

\section{Criteria for evaluation:}

Efficacy measurements

- The tumour morphologic response was evaluated by computed tomography scanner (CT-scan) using the revised Response Evaluation Criteria In Solid Tumors (RECIST) guideline v1.1 (Eisenhauer, 2009) at baseline within 30 days before the first S 78454 intake and every four cycles beginning Cycle 4, between D10 and D14. In case of response (complete response [CR], partial response [PR]), CT-scan (RECIST v1.1) was to be performed a month later for confirmation. An evaluation might have been performed at the withdrawal visit according to the investigators discretion. CT-scan was reviewed in the centre and by an independent central reviewer.

- The tumour metabolic response was assessed by 18-FluoroDeoxyGlucose-Positron Emission Tomography / Computed Tomography scanner ( $\left[{ }^{18}\right.$ FDG $]$-PET/CT-scan $)$ at baseline within 15 days before the first S 78454 intake and between D11 and D14 of Cycle 1. PET/CT-scan was reviewed by an independent central reviewer.

- Tumour markers in blood: carcinoembryonic antigen (CEA) and carbohydrate antigen 19-9 (CA 19-9), were assessed at the inclusion visit, on D1 (pre-dose) of all cycles, at the end of the study at the withdrawal visit.

\section{Safety measurements:}

- Recording of adverse events (AEs).

- Toxicity assessment according to National Cancer Institute Common Terminology Criteria for Adverse Events (NCI-CTCAE) version 3.0 (12 December 2003).

- Physical examination, ECOG status, weight, heart rate, blood pressure, neurological examination.

- Electrocardiogram (ECG). 


\section{Criteria for evaluation (Cont'd)}

\section{Safety measurements (Cont'd)}

- Laboratory tests:

- Haematology: haemoglobin, haematocrit, red blood cells (RBC) count, differential white blood cells (WBC) count, total WBC count, platelets count.

- Biochemistry: albumin, ionogram (sodium $[\mathrm{Na}]$, potassium $[\mathrm{K}]$, chlorine $[\mathrm{Cl}]$, calcium $[\mathrm{Ca}]$, magnesium $[\mathrm{Mg}]$, phosphate, bicarbonate), urea, serum creatinine (creatinine clearance), proteinemia, glycaemia, aspartate aminotransferase (AST), alanine aminotransferase (ALT), alkaline phosphatase (ALP), gamma glutamyltransferase (GGT), total bilirubin, lactate dehydrogenase (LDH). All samplings for biochemistry had to be taken in fasting conditions.

Pharmacokinetic measurements during Cycle 1:

Concentrations of S 78454 and its main metabolites were measured in plasma by liquid chromatography tandem mass spectrometry (LC-MS/MS) at D1, D2, D3 and D4 pre and post S 78454 doses.

Concentrations of oxaliplatin and 5-FU were measured in plasma at D3, D4 and D5.

Optional part: Pharmacogenetic measurement during Cycle 1

A pharmacogenetic analysis was performed in order to decipher the metabolism of S 78454 .

One blood sample was taken on Cycle 1 at Day 1, after S 78454 intake for this analysis.

\section{Statistical methods:}

Analysis Sets:

- Screened Set: This set corresponded to all screened patients.

- Included Set (IS): This set corresponded to all included patients.

- Safety Set (SS): This set corresponded to patients having taken at least one dose of study treatment.

- Full Analysis Set (FAS): Based on the intention-to-treat principle, this set corresponded to included patients who have taken at least one dose of study treatment and who have at least one baseline and one post-baseline tumour evaluation.

- DLT evaluable Set (DLTES): All patients from the SS who were evaluable for DLT according to the DLT assessment at end of Cycle 1. A patient was not considered evaluable if:

- $\mathrm{He} / \mathrm{she}$ discontinued during first cycle for a reason other than DLT or

- $\mathrm{He} / \mathrm{she}$ did not undergo a DLT assessment at the start of Cycle 2 or

$\mathrm{He} / \mathrm{she}$ did not receive at least six of eight doses of S 78454 unless treatment was stopped for DLT.

\section{Study outcome:}

Patients' status and protocol deviations were described by dose levels and overall in the IS. The main characteristics at baseline (demography, disease history and initial tumour assessment) were described at the RP2D and overall in the IS. Concomitant treatments (SS), treatment duration in patients (SS and FAS) and the extent of exposure during the treatment period (SS and FAS) were described at the RP2D and overall.

\section{Efficacy analysis:}

Efficacy analyses were carried out in the FAS. The best overall response (BOR) defined as the best response across all time points from the start of the study treatment until progressive disease (PD) or the end of the treatment period whichever came first, according to the investigators or the central review, were described by dose levels and overall. Complete response or PR were not confirmed following initial documentation. BOR over the whole study period excluded follow-up period. PFS defined as the time elapsed between the first drug intake and PD or death was provided at the RP2D and overall.

Safety analysis: Descriptive statistics were provided.

The safety analysis was assessed with a description of the DLTs on the DLTES, emergent adverse events (EAEs), serious adverse events (SAEs), death, laboratory parameters (biochemistry and haematology) using the NCI-CTCAE classification, vital signs (temperature, blood pressure, heart rate), ECOG performance status and ECG by dose levels and overall in the SS.

Pharmacokinetic analysis: Bioanalytical results are provided in separate reports in Appendix 16.4. 


\section{SUMMARY - CONCLUSIONS \\ DISPOSITION OF PATIENTS AND ANALYSIS SETS}

A total of 32 patients were included in the study. Patients received S 78454 p.o. treatment in combination with FOLFOX according to the amended protocol. All patients were withdrawn from the study mainly for progressive disease (22 patients, $68.8 \%$ ). The other reasons were AEs (six patients, $18.8 \%$ ), cure, remission or marked improvement (two patients, 6.3\%) or non-medical reasons (two patients, 6.3\%).

Disposition of patients - Included Set $(\mathrm{N}=32)$

S 78454

\begin{tabular}{|c|c|c|c|c|c|c|c|c|c|}
\hline & & $\begin{array}{c}80 \mathrm{mg} \\
\text { sBID } \\
(\mathrm{N}=3) \\
3\end{array}$ & $\begin{array}{c}100 \mathrm{mg} \\
\text { sBID } \\
(\mathrm{N}=3) \\
3\end{array}$ & $\begin{array}{c}120 \mathrm{mg} \\
\text { sBID } \\
(\mathrm{N}=4) \\
4\end{array}$ & $\begin{array}{c}140 \mathrm{mg} \\
\text { sBID } \\
(\mathrm{N}=4) \\
4\end{array}$ & $\begin{array}{c}140 \mathrm{mg} \\
\text { bBID } \\
(\mathrm{N}=6) \\
6\end{array}$ & $\begin{array}{c}160 \mathrm{mg} \\
\text { sBID } \\
(\mathrm{N}=6) \\
6\end{array}$ & $\begin{array}{c}160 \mathrm{mg} \\
\text { bBID } \\
(\mathrm{N}=6) \\
6\end{array}$ & $\begin{array}{c}\begin{array}{c}\text { All } \\
(\mathbf{N}=\mathbf{3 2})\end{array} \\
\mathbf{3 2}\end{array}$ \\
\hline & & 1 & - & & 1 & & 1 & & \\
\hline & & & - & $\ldots$ & $5.00)$ & - & 6.67) & - & 9.38) \\
\hline $\begin{array}{l}\text { With protocol deviation } \\
\text { before or at inclusion }\end{array}$ & $\begin{array}{l}\mathrm{n} \\
(\%)\end{array}$ & $\begin{array}{c}2 \\
(66.67)\end{array}$ & $\begin{array}{c}3 \\
(100)\end{array}$ & $\begin{array}{c}4 \\
(100)\end{array}$ & $\begin{array}{c}3 \\
(75.00)\end{array}$ & $\begin{array}{c}6 \\
(100)\end{array}$ & $\begin{array}{c}5 \\
(83.33)\end{array}$ & $\begin{array}{c}6 \\
(100)\end{array}$ & $\begin{array}{c}29 \\
(90.63)\end{array}$ \\
\hline
\end{tabular}

\begin{tabular}{llllcccccc} 
Withdrawn due to & Nobs & $\mathbf{3}$ & $\mathbf{3}$ & $\mathbf{4}$ & $\mathbf{4}$ & $\mathbf{6}$ & $\mathbf{6}$ & $\mathbf{6}$ & $\mathbf{3 2}$ \\
Adverse events & $\mathrm{n}$ & - & - & 2 & 1 & 1 & 2 & - & 6 \\
& $(\%)$ & - & - & $(50.00)$ & $(25.00)$ & $(16.67)$ & $(33.33)$ & - & $(18.75)$ \\
Progressive disease & $\mathrm{n}$ & 2 & 3 & 2 & 3 & 4 & 4 & 4 & $22^{*}$ \\
& $(\%)$ & $(66.67)$ & $(100)$ & $(50.00)$ & $(75.00)$ & $(66.67)$ & $(66.67)$ & $(66.67)$ & $(68.75)$ \\
\multirow{2}{*}{ Non-medical reasons } & $\mathrm{n}$ & - & - & - & - & - & - & 2 & 2 \\
\multirow{2}{*}{$\begin{array}{l}\text { Cure, remission or marked } \\
\text { improvement }\end{array}$} & $(\%)$ & - & - & - & - & - & - & $(33.33)$ & $(6.25)$ \\
\hline
\end{tabular}

sBID: $S 78454$ treatment expressed in salt according to the initial protocol, twice a day.

bBID: $S 78454$ treatment expressed in base according to the amended protocol, twice a day.

$n:$ Number of patients.

$N$ : Number of patients in each analysis sets.

$\%:(n / N) * 100$.

* of note one of the patients withdrawn for progressive disease had an AE malignant neoplasm progression with action taken drug withdrawn (patient 006380020160022).

Analysis Sets

\section{S 78454}

\begin{tabular}{|c|c|c|c|c|c|c|c|c|c|}
\hline & & $\begin{array}{l}\text { 80mg } \\
\text { sBID }\end{array}$ & $\begin{array}{l}100 \mathrm{mg} \\
\text { sBID }\end{array}$ & $\begin{array}{c}120 \mathrm{mg} \\
\text { SBID }\end{array}$ & $\begin{array}{l}140 \mathrm{mg} \\
\text { sBID }\end{array}$ & $\begin{array}{l}140 \mathrm{mg} \\
\text { bBID }\end{array}$ & $\begin{array}{l}160 \mathrm{mg} \\
\text { sBID }\end{array}$ & $\begin{array}{l}160 \mathrm{mg} \\
\text { bBID }\end{array}$ & $\underset{\text { Not }}{\text { Noated }}$ \\
\hline Screened Set $(N=35)$ & $\begin{array}{l}\mathrm{n} \\
(\%)\end{array}$ & $\begin{array}{c}3 \\
(8.6)\end{array}$ & $\begin{array}{c}3 \\
(8.6)\end{array}$ & $\begin{array}{c}4 \\
(11.4)\end{array}$ & $\begin{array}{c}4 \\
(11.4)\end{array}$ & $\begin{array}{c}6 \\
(17.1)\end{array}$ & $\begin{array}{c}6 \\
(17.1)\end{array}$ & $\begin{array}{c}6 \\
(17.1)\end{array}$ & $\begin{array}{c}3 \\
(8.6)\end{array}$ \\
\hline Included Set $(\mathrm{N}=32)$ & $\begin{array}{l}\mathrm{n} \\
(\%)\end{array}$ & $\begin{array}{c}3 \\
(9.4)\end{array}$ & $\begin{array}{c}3 \\
(9.4)\end{array}$ & $\begin{array}{c}4 \\
(12.5)\end{array}$ & $\begin{array}{c}4 \\
(12.5)\end{array}$ & $\begin{array}{c}6 \\
(18.8)\end{array}$ & $\begin{array}{c}6 \\
(18.8)\end{array}$ & $\begin{array}{c}6 \\
(18.8)\end{array}$ & $\begin{array}{l}- \\
-\end{array}$ \\
\hline $\begin{array}{l}\text { DLT Evaluable Set } \\
(\mathrm{N}=23)\end{array}$ & $\begin{array}{l}\mathrm{n} \\
(\%)\end{array}$ & $\begin{array}{c}3 \\
(13.0)\end{array}$ & $\begin{array}{c}3 \\
(13.0)\end{array}$ & $\begin{array}{c}3 \\
(13.0)\end{array}$ & $\begin{array}{c}3 \\
(13.0)\end{array}$ & NA & $\begin{array}{c}6 \\
(26.1)\end{array}$ & $\begin{array}{c}5 \\
(21.7)\end{array}$ & $\begin{array}{l}- \\
-\end{array}$ \\
\hline $\begin{array}{l}\text { Full Analysis Set } \\
(\mathrm{N}=22)\end{array}$ & $\begin{array}{l}\mathrm{n} \\
(\%)\end{array}$ & $\begin{array}{c}3 \\
(13.6)\end{array}$ & $\begin{array}{c}2 \\
(9.1)\end{array}$ & $\begin{array}{c}2 \\
(9.1)\end{array}$ & $\begin{array}{c}2 \\
(9.1)\end{array}$ & $\begin{array}{c}5 \\
(22.7)\end{array}$ & $\begin{array}{c}4 \\
(18.2)\end{array}$ & $\begin{array}{c}4 \\
(18.2)\end{array}$ & $\begin{array}{l}- \\
-\end{array}$ \\
\hline Safety Set $(\mathrm{N}=32)$ & $\begin{array}{l}\mathrm{n} \\
(\%)\end{array}$ & $\begin{array}{c}3 \\
(9.4)\end{array}$ & $\begin{array}{c}3 \\
(9.4)\end{array}$ & $\begin{array}{c}4 \\
(12.5)\end{array}$ & $\begin{array}{c}4 \\
(12.5)\end{array}$ & $\begin{array}{c}6 \\
(18.8)\end{array}$ & $\begin{array}{c}6 \\
(18.8)\end{array}$ & $\begin{array}{c}6 \\
(18.8)\end{array}$ & $\begin{array}{l}- \\
-\end{array}$ \\
\hline
\end{tabular}

SBID: $S 78454$ treatment expressed in salt according to the initial protocol, twice a day.

bBID: $S 78454$ treatment expressed in base according to the amended protocol, twice a day.

$n:$ Number of patients.

$N$ : Number of patients in each analysis sets.

$\%:(n / N) * 100$. 


\section{SUMMARY - CONCLUSIONS (Cont'd) \\ BASELINE CHARACTERISTICS}

Demographic and baseline characteristics of patients in the Included Set $(\mathrm{N}=32)$ were in line with inclusion criteria defined for the study. The patients had a mean age of $57 \pm 11$ years old (ranging from 25 to 74 years). Most patients (65.6\%) were male.

At inclusion in the study, all patients had a solid tumour since a mean of $3.0 \pm 2.6$ years (median $=2.4$ years). Overall, the patients had mostly a colon adenocarcinoma with 17 patients $(53.1 \%)$. The other solid tumours were gastric adenocarcinoma with four patients $(12.5 \%)$, rectal adenocarcinoma with four patients $(12.5 \%)$, colorectal adenocarcinoma with three patients $(9.4 \%)$, pancreatic adenocarcinoma with three patients $(9.4 \%)$, and malignant neoplasm of the pancreatic duct with one patient (3.1\%).

At the time of diagnosis, patients had tumour stages T3 for 17 patients $(54.8 \%)$, T4 for eight patients $(25.8 \%)$ or T2 for five patients $(16.1 \%)$ overall, according to the TNM classification. Regarding the extension of the cancer, 13 patients $(41.9 \%)$ overall had tumours spread to the lymph nodes (seven [22.6\%] to regional lymph nodes and six [19.4\%] to distant lymph nodes) and 20 patients $(64.5 \%)$ overall had distant metastasis. One patient had a tumour classified Stage D (distant metastasis) using the former Duke's Classification modified by Astler-Coller. Tumour stage assessment was missing for one patient.

At inclusion, 21 patients $(65.6 \%)$ were in relapse and all patients had metastasis consequent to their disease evolution.

\section{EXTENT OF EXPOSURE}

In the Safety Set $(\mathrm{N}=32)$, the mean duration $( \pm \mathrm{SD})$ of the treatment was $9.5( \pm 7.9)$ weeks (median: 7.5 weeks) ranging from 2.0 to 40.0 weeks. The patients received on average $4.3( \pm 3.5)$ cycles (median: 3.5 cycles). The mean relative dose intensity per patient (i.e. compliance) $( \pm \mathrm{SD})$ was $85.8 \pm 16.1 \%$ and the mean cumulative dose received by patient $( \pm \mathrm{SD})$ was $4527 \pm 4046 \mathrm{mg}$.

In the Full Analysis Set $(\mathrm{N}=22)$, the mean duration $( \pm \mathrm{SD})$ of the treatment was $11.5( \pm 8.8)$ weeks (median: 9.0 weeks) ranging from 2.0 to 40.0 weeks. The patients received on average $( \pm \mathrm{SD}) 5.2( \pm 3.9)$ cycles (median: 4.0 cycles). The mean relative dose intensity per patient (i.e. compliance) ( $\pm \mathrm{SD}$ ) was $86.6 \pm 13.1 \%$ and the mean cumulative dose received by patient $( \pm \mathrm{SD})$ was $5483 \pm 4557 \mathrm{mg}$.

As the MTD was not reached during the four dose levels planned in the protocol, additional cohorts were added in accordance with the protocol: $160 \mathrm{mg}$ b.i.d. expressed in salt (160 $\mathrm{mg} \mathrm{S}$ b.i.d.) and $160 \mathrm{mg}$ b.i.d expressed in base (160 $\mathrm{mg} \mathrm{B}$ b.i.d., following the change in the manufacturing process). MTD was determined at $160 \mathrm{mg}$ B b.i.d. As RP2D was defined as one dose step below MTD, RP2D was set at $140 \mathrm{mg} \mathrm{B}$ b.i.d. Six additional patients were included at this dose in the confirmatory part. Finally, three patients were treated at $80 \mathrm{mg} \mathrm{S}$ b.i.d., three patients at $100 \mathrm{mg} \mathrm{S} \mathrm{b.i.d.,} \mathrm{four} \mathrm{patients} \mathrm{at} 120 \mathrm{mg} \mathrm{S}$ b.i.d., four patients at $140 \mathrm{mg} \mathrm{S}$ b.i.d., six patients at $140 \mathrm{mg} \mathrm{B}$ b.i.d., six patients at $160 \mathrm{mg} \mathrm{S}$ b.i.d. and six patients at $160 \mathrm{mg} \mathrm{B}$ b.i.d..

\section{EFFICACY RESULTS}

Among the 22 patients co-treated with S 78454 and FOLFOX in the FAS, the BOR was stable disease (SD) in eight patients (36.4\%) or PD in 13 patients $(59.1 \%)$. The evaluation was missing for one patient treated at the RP2D.

The clinical benefit of the treatment was evaluated using PFS. One patient was censored for being alive without new treatment or PD. The 21 remaining patients had all PD. The median PFS was 8.5 weeks overall (21 patients) and 7.6 weeks at the RP2D (four patients). 


\section{SUMMARY - CONCLUSIONS (Cont'd) \\ SAFETY RESULTS}

\section{- Emergent adverse events and DLTs}

Summary of safety results (according to the investigators)

\begin{tabular}{|c|c|c|}
\hline & & All $(\mathbf{N}=32)$ \\
\hline \multicolumn{3}{|l|}{ Patients having reported at least one } \\
\hline EAE & $\mathrm{n}(\%)$ & $32(100)$ \\
\hline Treatment-related EAE & $\mathrm{n}(\%)$ & $29(90.6)$ \\
\hline \multicolumn{3}{|l|}{ Patients having reported at least one } \\
\hline Emergent SAE & $\mathrm{n}(\%)$ & $14(43.8)$ \\
\hline Treatment-related emergent SAE & $\mathrm{n}(\%)$ & $6(18.8)$ \\
\hline \multicolumn{3}{|l|}{ Patients who discontinue the treatment due to } \\
\hline EAE & $\mathrm{n}(\%)$ & $5^{*}(15.6)$ \\
\hline Emergent SAE & $\mathrm{n}(\%)$ & $3(9.4)$ \\
\hline Treatment-related EAE & $\mathrm{n}(\%)$ & $2(6.3)$ \\
\hline Treatment-related emergent SAE & $\mathrm{n}(\%)$ & - \\
\hline Patients who died during the treatment period & $\mathrm{n}(\%)$ & 1 \\
\hline Patients who died during the follow-up period & $\mathrm{n}(\%)$ & 9 \\
\hline
\end{tabular}

During the study, all patients reported at least one EAE for a total of 639 EAEs. The most frequently affected system organ classes (SOCs) were gastrointestinal disorders (27 patients, 84.4\%), blood and lymphatic system disorders (25 patients, 78.1\%), and general disorders and administration site conditions (21 patients, 65.6\%). The most frequently reported EAEs were thrombocytopenia (20 patients, 62.5\%), diarrhoea (17 patients, $53.1 \%$ ) and anaemia (17 patients, 53.1\%).

Most of the EAEs resolved or were improving (81.8\%) at the end of the study.

One patient treated at $160 \mathrm{mg} \mathrm{S}$ b.i.d. died during the treatment period. Two Grade 5 emergent SAEs were reported for this patient including metastases to the mediastinum and diaphragmatic paralysis both considered as not related to the study treatment.

EAEs reported as treatment-related according to investigators' opinion occurred in 29 patients $(90.6 \%)$. They were mostly thrombocytopenia (20 patients, $62.5 \%$, including one patient having Grade 4 thrombocytopenia as DLT), vomiting (10 patients, 31.3\%), nausea (nine patients, 28.1\%), anaemia (nine patients, 28.1\%), neutropenia (eight patients, 25.0\%), diarrhoea (six patients, 18.8\%), asthenia (five patients, 15.6\%), electrocardiogram QT prolonged (four patients, $12.5 \%$ ) or fatigue (four patients, $12.5 \%$ ).

EAEs (including emergent SAEs) led to the study treatment discontinuation in five patients. Two of these EAEs were considered by the investigators as related to S 78454: a Grade 3 electrocardiogram QT prolonged in one patient treated at $140 \mathrm{mg} \mathrm{B}$ b.i.d. and a Grade 3 hyperbilirubinaemia in one patient treated at $160 \mathrm{mg}$ S b.i.d.

At the end of Cycle 1, the following AEs were considered as DLTs:

- Grade 3 hyperbilirubinaemia in one patient treated at $160 \mathrm{mg} \mathrm{S}$ b.i.d.

- Grade 4 thrombocytopenia in one patient treated at $160 \mathrm{mg} \mathrm{B}$ b.i.d.

The dose of $160 \mathrm{mg} \mathrm{B}$ b.i.d. was considered as the MTD and $140 \mathrm{mg}$ B b.i.d. as the RP2D.

Emergent SAEs according to the investigator were reported in 14 patients (43.8\%) for 31 events. The most frequently reported emergent SAEs were thrombocytopenia (four patients, 12.5\%), malignant neoplasm progression (four patients, 12.5\%), dysphagia (two patients, 6.3\%), subileus (two patients, 6.3\%) and asthenia (two patients, 6.3\%). Seven of these events were considered as treatment-related: four thrombocytopenia including the Grade 4 thrombocytopenia considered as DLT, one anaemia, one sinus bradycardia and one hyperbilirubinaemia. Three emergent SAEs led to the study treatment withdrawal: subileus, malignant neoplasm progression and renal failure acute. None of these emergent SAEs (according to the investigator) leading to S 78454 withdrawal have been related to S 78454 by the investigators. 


\section{SUMMARY - CONCLUSIONS (Cont'd) \\ SAFETY RESULTS (Cont'd)}

In addition, the Sponsor upgraded 9 AEs in 8 patients as serious AEs: two grade 4 thrombocytopeniae, one grade 4 neutropenia, one grade 3 hypokalaemia, one grade 4 hypokalaemia, one grade $3 \mathrm{QTcF}$ prolongation (leading to drug withdrawal), two episodes of grade 2 HTA in one patient, and one grade 3 hyperglycaemia.

A total of 10 deaths were reported during the study. In addition to the patient who died during the treatment period, nine patients died during the period following the treatment period: seven from progressive disease and two from unspecified reasons.

\section{- Laboratory tests: biochemistry}

The worst values during the treatment period were analysed on a selection of main parameters (ALT, AST, ALP, albumin, total bilirubin and creatinine clearance). Regarding the biochemistry parameters rated according to the CTCAE grading overall, 11 Grade 3 abnormal values were reported in seven patients for transaminases, ALP, albumin or total bilirubin. The two severe abnormal values for increase of ALT (Grade 3) were reported as alanine aminotransferase increase $\mathrm{AE}$ by the investigators. One of the two severe abnormal values for increase of AST (Grade 3) was reported as aspartate aminotransferase increase AE by the investigator. One of the two severe abnormal values for increase of ALP (Grade 3) was reported as blood alkaline phosphatase increase AE by the investigator. One of the two severe abnormal values for decrease of albumin (Grade 3) was reported as hypoalbuminaemia $\mathrm{AE}$ by the investigator. One of the three severe abnormal values for increase of total bilirubin (Grade 3) was reported as hyperbilirubinaemia AE by the investigator. No out-of-range value for creatinine clearance, which is a non-gradable parameter according to CTCAE grading, was observed.

\section{- Laboratory tests: haematology}

Regarding the haematological parameters rated according to the CTCAE grading, emergent severe abnormal values were detected for low amount of the following parameters:

- Haemoglobin: four patients graded 3 (one patient treated at $80 \mathrm{mg} \mathrm{S}$ b.i.d., one patient treated at $120 \mathrm{mg}$ $\mathrm{S}$ b.i.d., one patient treated at $140 \mathrm{mg} \mathrm{S}$ b.i.d. and one patient treated at $160 \mathrm{mg} \mathrm{B}$ b.i.d.).

- WBC: two patients graded 3 (one patient treated at $120 \mathrm{mg} \mathrm{S}$ b.i.d. and one patient treated at $140 \mathrm{mg} \mathrm{S}$ b.i.d.).

- Neutrophils: seven patients graded 3 (one patient treated at $80 \mathrm{mg} \mathrm{S}$ b.i.d., one patient treated at $120 \mathrm{mg}$ $\mathrm{S}$ b.i.d., three patients treated at $140 \mathrm{mg} \mathrm{S}$ b.i.d., one patient treated at $140 \mathrm{mg} \mathrm{B}$ b.i.d. and one patient treated at $160 \mathrm{mg} \mathrm{S}$ b.i.d.).

- Lymphocytes: six patients graded 3 (two patients treated at $120 \mathrm{mg} \mathrm{S}$ b.i.d., one patient treated at $140 \mathrm{mg} \mathrm{S}$ b.i.d., one patient treated at $160 \mathrm{mg} \mathrm{S} \mathrm{b.i.d.} \mathrm{and} \mathrm{two} \mathrm{patients} \mathrm{treated} \mathrm{at} 160 \mathrm{mg} \mathrm{B} \mathrm{b.i.d.)} \mathrm{and}$ two patients graded 4 (one patient treated at $120 \mathrm{mg} \mathrm{S}$ b.i.d. and, one patient treated at $160 \mathrm{mg} \mathrm{S}$ b.i.d.).

- Platelets: five patients graded 3 (one patient treated at $80 \mathrm{mg} \mathrm{S}$ b.i.d., one patient treated at $120 \mathrm{mg} \mathrm{S}$ b.i.d., one patient treated at $140 \mathrm{mg} \mathrm{S}$ b.i.d., one patient treated at $160 \mathrm{mg} \mathrm{S}$ b.i.d. and one patient treated at $160 \mathrm{mg} \mathrm{B} \mathrm{b.i.d.)} \mathrm{and} \mathrm{four} \mathrm{patients} \mathrm{graded} 4$ (one patient treated at $80 \mathrm{mg} \mathrm{S}$ b.i.d., one patient treated at $120 \mathrm{mg} \mathrm{S}$ b.i.d., one patient treated at $140 \mathrm{mg} \mathrm{S}$ b.i.d. and one patient treated at $160 \mathrm{mg} \mathrm{B}$ b.i.d.).

Among these patients, a Grade 4 thrombocytopenia was reported and considered as a DLT in one patient.

\section{- Other safety evaluation}

According to ECG central reading, emergent ECG abnormalities reported as EAE included nine electrocardiogram QT prolonged in four patients (12.5\%), two electrocardiogram QRS complex prolonged in one patient $(3.1 \%)$, one electrocardiogram $\mathrm{T}$ wave amplitude decreased in one patient $(3.1 \%)$, one electrocardiogram $\mathrm{T}$ wave inversion in one patient (3.1\%), and one electrocardiogram repolarization abnormality in one patient $(3.1 \%)$.

Regarding QTc Fridericia interval (QTcF), one patient treated at $140 \mathrm{mg} \mathrm{S}$ b.i.d. presented with a maximum absolute QTcF interval value $>500 \mathrm{~ms}$ and a maximum QTcF interval change from baseline $>60 \mathrm{~ms}$. Five patients presented with a maximum absolute QTcF interval value between 450 and $480 \mathrm{~ms}$ and one patient with a maximum absolute QTcF interval during the treatment period value between 480 and $500 \mathrm{~ms}$. Twelve patients presented with a maximum QTcF interval change from baseline between 30 and $60 \mathrm{~ms}$ and three patients with a maximum QTcF change beyond $60 \mathrm{~ms}$. 


\section{CONCLUSION}

In this open dose-escalation Phase I study with doses of S 78454 (abexinostat) p.o. administered in combination with FOLFOX, a total of 32 patients were included, 32 received the study treatment, and 22 were evaluable for the response. The scheme of the study was administration of $\mathbf{S} 78454$ b.i.d. for four consecutive days (D1-4) during a 2-week cycle with infusion of FOLFOX on D3-5 of each cycle. The maximum tolerated dose (MTD) was determined at $160 \mathrm{mg}$ b.i.d. (expressed in base) and the recommended dose at $140 \mathrm{mg}$ b.i.d. (expressed in base). Two DLTs were reported: one Grade 3 hyperbilirubinaemia in one patient treated at $160 \mathrm{mg}$ b.i.d. (expressed in salt) and one Grade 4 thrombocytopenia in one patient treated at $160 \mathrm{mg}$ b.i.d. (expressed in base).

Concerning evaluation of clinical activity, eight patients had stable disease as best overall response. Thirteen other patients had progressive disease. One patient was not evaluated.

The most frequent emergent adverse events considered treatment-related by the investigators were thrombocytopenia, vomiting, nausea, anaemia, neutropenia, diarrhoea, asthenia, electrocardiogram QT prolonged or fatigue.

In the present trial, the safety profile of abexinostat in combination with FOLFOX using the studied administration schedule was acceptable and consistent with our knowledge of the drugs.

Date of the report: 19 May 2015

Version of the report: Final version 